\title{
Measurement and feedback for cooling heavy levitated particles in low-frequency traps
}

\author{
L. S. Walker, G. R. M. Robb, and A. J. Daley \\ Department of Physics and SUPA, University of Strathclyde, 107 Rottenrow, Glasgow G4 ONG, United Kingdom
}

(Received 26 March 2019; published 12 December 2019)

\begin{abstract}
We consider a possible route to ground-state cooling of a levitated nanoparticle, magnetically trapped by a strong permanent magnet, using a combination of measurement and feedback. The trap frequency of this system is much lower than those involving trapped ions or nanomechanical resonators. Minimization of environmental heating is therefore challenging as it requires control of the system on a timescale comparable to the inverse of the trap frequency. We show that these traps are an excellent platform for performing optimal feedback control via real-time state estimation, for the preparation of motional states with measurable quantum properties.
\end{abstract}

DOI: 10.1103/PhysRevA.100.063819

\section{INTRODUCTION}

The ability to prepare and manipulate quantum states of nanomechanical systems is of interest in metrology and for tests of fundamental quantum physics. Ground-state cooling has already been achieved in cryogenic chambers with silicon membranes and other microwave devices [1,2]. However, there is a desire to produce quantum states of motion with levitated particles that are not physically tethered to their surroundings, and which therefore have significantly longer decoherence times. If realized, these systems would be a platform for many novel experiments: tests of wave-function collapse models [3], for ultrasensitive metrology [4], and to probe gravitational decoherence [5]. Most of the progress towards preparing ground-state systems has been made with optically levitated particles, where recent experiments are currently capable of detecting - and are limited by-photon shot noise [6].

Although optical traps are the most widely used for trapping microscopic particles, they can face problems with heating due to the high laser intensities [7] and the intrinsic noise associated with the trapping force. While optical traps produce oscillators with excellent quality factors, there are alternatives that circumvent some of their current limitations. Static magnetic traps are free from intrinsic heating due to confinement, and have recently been demonstrated as suitable for trapping and cooling nanodiamonds [8,9]. The traps are typically three orders of magnitude larger than their optical counterparts and consequently operate at much lower frequencies, of around $100 \mathrm{~Hz}$ as opposed to $100 \mathrm{kHz}$ for an optical trap. This comes with the advantage of being able to hold and manipulate large particles, but also makes it unfeasible to cool on timescales much longer than the oscillation period because of their relatively low quality factor. Although the oscillation frequency is significantly lower than in an optical trap, magnetic confinement is subject to similar environmental heating. Current experiments $[6,8]$ have estimated the phonon reheating rate for these systems in high vacuum $\left(10^{-8}\right.$ mbar $)$ to be $\Gamma_{\text {th }} \approx 100 \mathrm{~Hz}$ and it is expected that this will be significantly reduced at lower pressures.
In this article, we consider methods for improving the quantum measurement efficiency of levitated nanoparticles, and go on to analyze how best to apply feedback and assess the fundamental cooling limits. Direct feedback of a position measurement in the form developed by Wiseman and Milburn [10] has been shown to be effective in controlling the motion of optically levitated ions [11], but we find it to be less suitable here. The cooling strategies employed with direct feedback rely heavily on a separation of timescales between the damping and trap frequency that is impractical in larger traps. Instead, our starting point is to adapt the real-time state estimation and the feedback strategies discussed by Doherty et al. [12] for use in this newly accessible low-frequency regime.

Having considered several options for tracking a particle's position and momentum, we suggest making measurements in two steps. At first, scattered light from the particle can be imaged with a quadrant photodiode, and an externally applied damping force can be used for cooling. After damping the particle's motion to sub-optical-wavelength amplitudes, significantly better resolution can be achieved by measuring how the particle scatters light into the mode of an adjacent single mirror for improved detection efficiency. Continuous feedback can be applied by simultaneously shifting the trap center and by applying an external damping force via magnetic or optical fields. We go on to show that the proposed methods could be used to produce motional states of microscopic oscillators with average phonon occupancy $\langle n\rangle<3$ and state purity $P \approx 0.44$, achievable with realistic measurement efficiencies for current experiments. This is a regime where it should already be possible to see signs of quantum behavior in the particle motion, and could provide a starting point for preparation of more exotic macroscopic superposition states. An ideal candidate particle for future experiments would be an approximately spherical nanodiamond, which is of interest due to access to internal nitrogen vacancies $(\mathrm{NV})$. This second quantum handle on the particle is crucial for many proposed future experiments [3,5] and may also provide a route to having fine control over micron-sized, as opposed to nanometer-sized, particles. With advances in isolation from environmental heating and improvements in 
light-collection efficiency, there are no fundamental limits to these techniques being used to reach the quantum motional ground state.

The rest of this article is organized as follows. In Sec. II, we review the stochastic master equation that results from measuring the motion of a particle in front of a single mirror. In Sec. III, we discuss the merits and limitations of various measurement schemes and the practicality of real-time state estimation. In Sec. IV, we show the effectiveness of feedback by estimation, in cooling and squeezing mechanical motion. We conclude and present outlooks in Sec. V.

\section{MODEL}

Levitated, trapped particles for the purpose of cooling are, by design, simple oscillators. Our model describes the motion of a magnetically confined particle and its interaction with an optical probe beam. Magnetic confinement provides large trapping potentials capable of holding very massive particles, with the potential to explore truly macroscopic quantum objects. We will, however, focus on smaller particles, tens of nanometers in size, for their simple light-scattering properties. Nanoparticles can be treated as point dipoles and their motion is simpler to measure at sub-optical wavelengths. We will treat the internal dynamics of the light-scattering process adiabatically and model the particle as a point dipole in the Rayleigh regime. Further consideration of larger particles is given in the conclusions. The Hamiltonians of the freely oscillating particle, $H_{\text {sys }}$, the optical field, $H_{\mathrm{F}}$, and the interaction Hamiltonian, $H_{\mathrm{I}}$, are given by

$$
\begin{gathered}
H_{\mathrm{sys}}=\frac{p^{2}}{2 m}+\frac{m \omega^{2} x^{2}}{2}, \\
H_{\mathrm{F}}=\sum_{k} \hbar \omega_{k} b_{k}^{\dagger} b_{k}, \\
H_{\mathrm{I}}=\sum_{k} \hbar \sqrt{\gamma}\left[b_{k} \exp (i \mathbf{k} \cdot \mathbf{r})+b_{k}^{\dagger} \exp (-i \mathbf{k} \cdot \mathbf{r})\right],
\end{gathered}
$$

where $m$ is the particle mass, $\omega$ is the magnetic trap frequency, $\gamma$ is the scattering rate into each mode of the optical electric field, and $b_{k}\left(b_{k}^{\dagger}\right)$ is the usual quantized field mode amplitude, with wave number and angular frequency of $k, \omega_{k}$, respectively. The momentum recoil due to the scattered photons is represented by $\mathbf{k} \cdot \mathbf{r}$, where $\mathbf{r}$ is the particle's position. It is sufficient to model the motion of the particle in one dimension (1D), as although some cooling is often applied along each trap axis, the frequencies of each motional degree of freedom can be well separated and safely decoupled, as is done in current experiments [8].

Continuous-measurement theory allows for quantification of the disturbance caused to the particle in relation to the amount of position information carried away by the field [13]. We will go on to discuss the merits and drawbacks of various measurement schemes, but first we outline the details of the method we assess to be the most suitable for magnetically levitated particles.

\section{Motional sideband detection}

The setup we consider uses a mirror to introduce a standing-wave mode across the levitated particle, where some

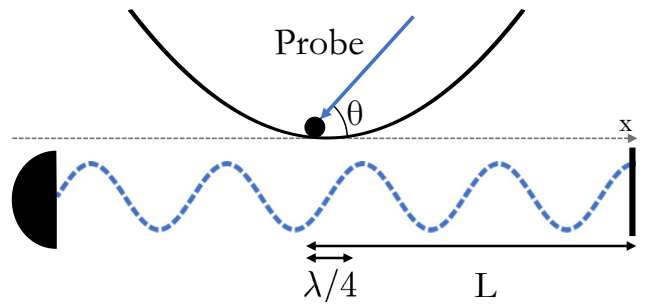

FIG. 1. Sketch of apparatus for measuring the intensity of a standing wave, modulated by a particle's motion along its main trap axis $x$. The trap center is marked a distance $L$ from the mirror, with the probe light incident at an angle $\theta$. The range of motion over which this measurement would be valid is restricted about a node of the standing light field, and has also been marked. A focused beam and a curved mirror that maximizes coupling into the reflected mode could reasonably produce light-collection efficiency $>15 \%$.

of the scattered light from the illumination probe will be collected, as shown schematically in Fig. 1. The mirrors here can be quite large, capturing a significant fraction of the light scattered along the primary trap axis. The particle motion adds sidebands to the spectrum of light scattered in the mirror mode, positioned at $\pm \omega$ from the optical frequency. Continuous measurement of these sidebands can be used to infer the particle's current position after filtering out the elastically scattered signal. This is a nonintrusive setup that could be implemented in magnetic traps to give a significant increase in measurement efficiency and resulting position resolution, over current imaging schemes.

The interaction Hamiltonian, considering only emission into the mirror mode, is

$$
H_{\mathrm{I}}=\hbar \sqrt{\gamma} \sin \left[k_{L}(L+\hat{x})\right]\left(b+b^{\dagger}\right) .
$$

If the position of the trap center is taken to be where $k_{L} L=$ $\pi / 4$, we can define the corresponding system operator,

$$
\hat{c}=\sin \left[k_{L}(L+\hat{x})\right] \approx \frac{1}{\sqrt{2}}\left(1+k_{L} \hat{x}\right),
$$

where we have performed a Taylor expansion in the LambDicke regime. This expansion is possible when the typical length of the oscillation is small compared with the wavelength of incident light, $k_{L} x \ll 1$ (some initial cooling would be required to reach this regime). We note that this operator has two separate components, describing the effects of constant amplitude elastically scattered light and positiondependent modulated light.

We can then apply continuous-measurement theory from quantum optics $[14,15]$ to the system. Under the usual Born and Markov approximations, for this form of the interaction Hamiltonian, we can think of the operator $\hat{c}$ as being applied to the system whenever a photon is emitted into the field $\rho \rightarrow \hat{c} \rho \hat{c}^{\dagger} /\left\langle\hat{c}^{\dagger} \hat{c}\right\rangle$. A stochastic increment $d N$ can be used to model whether or not a photon is detected in a given time step in the environment, taking a value of zero or one, respectively. Imagining for now that all the scattered light is somehow collected in the mirror mode, its average value should be the detection rate,

$$
\langle d N\rangle=\gamma\left\langle\hat{c}^{\dagger} \hat{c}\right\rangle d t
$$


corresponding to the expected value of measuring a scattered photon in the mirror mode in a time interval $d t$. In the limit where the component of elastically scattered light is comparatively large, it is helpful to make a diffusion approximation, as is commonly done when considering homodyne detection [16]. This is indeed the case here and so

$$
\begin{aligned}
d N & =\gamma \hat{c}^{\dagger} \hat{c} d t \approx \frac{\gamma}{2} d t+\gamma k_{L} \hat{x} d t \\
& =\frac{\gamma}{2} d t+\gamma k_{L}\langle x\rangle d t+\frac{\sqrt{\gamma}}{2} d W,
\end{aligned}
$$

where in the last line we have followed the usual analysis for random events occurring quickly enough to be treated as continuous noise, splitting the increment on the right-hand side into a sum of two parts: one deterministic and the other stochastic. The Wiener increment $d W$ represents Gaussian white noise. This signal corresponds directly to what would be measured experimentally by a photo detector. The resulting state evolution is described by a master equation conditioned on the Gaussian measurement collapse [13],

$$
d \rho=-\frac{i}{\hbar}\left[H_{\text {sys }}, \rho\right] d t+2 \kappa \mathcal{D}[x] \rho d t+\sqrt{2 \eta \kappa} \mathcal{H}[x] \rho d W .
$$

Here, $\mathcal{D}[x]$ is the usual Lindblad superoperator that describes dissipation and $\mathcal{H}[x]$ is the measurement superoperator that localizes the particle based on the information gathered,

$$
\begin{gathered}
\mathcal{D}[\hat{c}] \rho=\hat{c} \rho \hat{c}^{\dagger}-\frac{1}{2}\left(\hat{c}^{\dagger} \hat{c} \rho+\rho \hat{c}^{\dagger} \hat{c}\right), \\
\mathcal{H}[\hat{c}] \rho=\hat{c} \rho+\rho \hat{c}^{\dagger}-\left\langle\hat{c}+\hat{c}^{\dagger}\right\rangle \rho .
\end{gathered}
$$

The measurement strength $\kappa$ is defined as the ratio between the scattering rate and the reduction in position uncertainty of the particle due to each photon, which ideally corresponds to localization to within an optical wavelength,

$$
\kappa=\frac{\gamma k_{L}^{2}}{2} .
$$

This measurement strength reflects the rate of information gained about the system and the corresponding disturbance this necessarily causes. This exact expression for $\kappa$ would be accurate if the scattering was exclusively along the $x$ axis; the true value will be less in any other case where we should only count the momentum kicks projected along the $x$ direction. This is a small correction and should not be a problem given that $\kappa$ otherwise scales with increasing scattering rate off the particle and can be adjusted by increasing the laser power. The parameter $\eta$ is the quantum efficiency and accounts for the fraction of photons collected (after projection along the measurement axis) and any further loss that occurs in the detector. The measured photocurrent can be expressed as a renormalization of the now continuous photon count $\langle d N\rangle$, after subtracting the elastically scattered signal in postprocessing,

$$
d I=\langle x\rangle d t+\frac{1}{\sqrt{8 \eta \kappa}} d W .
$$

It has been suggested that light-collection efficiency of $\eta \approx 0.15$ could be reasonably expected when monitoring an optically trapped ion in front of a mirror [11]. One of the significant advantages of magnetic levitation is that the illumination light is independent of the trapping mechanism, which allows it to be shaped to optimize detection efficiency. This is of crucial importance when relying on active feedback cooling in order to counteract the random motion induced by the measurement itself. The shot noise in optically trapped nanoparticle experiments currently poses a major obstacle to reaching the ground state, with typical collection efficiencies $\eta<0.01$ [6].

\section{MEASUREMENT AND STATE ESTIMATION}

\section{A. Measurement}

The main obstacles to ground-state cooling using active feedback are environmental heating mechanisms and the fundamental disturbance associated with making measurements. In order to reach the quantum regime, it will be necessary for environmental heating to be made negligibly small on the timescales of the measurement and feedback. A reasonable goal in a magnetic trap would be to cool a particle in a time comparable to the oscillation period of a $\omega=2 \pi \times 100 \mathrm{~Hz}$ trap. In this case the phonon reheating rate would need to be reduced to around $\Gamma_{\mathrm{th}}=k_{B} T \gamma_{\mathrm{th}} / \hbar \omega \sim 1 \mathrm{~Hz}$, where $T$ represents the surrounding gas temperature and $\gamma_{\text {th }}$ is the thermal damping rate. Current typical reheating values are around $100 \mathrm{~Hz}$ and, below $10 \mathrm{mbar}$, thermal decoherence is expected to be linear in gas pressure and in the temperature of the environment. By better isolating the particle, or with the help of cryogenically cooling the trap chamber, reheating rates two orders of magnitude lower could feasibly be reached. Attempting to cool on timescales much shorter than the trap period has its own physical limitations. Fundamentally, this requires a stronger measurement, causing unavoidable heating through momentum diffusion, which we go on to analyze in detail.

It is helpful to consider the necessary measurement strength to reach a desired position resolution in a given time. A simple estimate of the resolution achievable across an interval $\Delta t$ can be found be integrating the measurement record [17],

$$
\Delta I=\int_{t}^{t+\Delta t} d I \approx\langle x\rangle \Delta t+\int_{t}^{t+\Delta t} \frac{d W}{\sqrt{8 \eta \kappa}} .
$$

In this expression, we have assumed that the expected value of the position of the particle will not change much over the time interval. This is not a well-justified assumption, but will allow us to determine an upper bound for the resolution. The integrated measurement signal $\Delta I$ has a mean value of $\sqrt{8 \eta \kappa}\langle x\rangle \Delta t$ that grows linearly in time, and its width grows as the square root, $\sigma=\sqrt{\Delta t}$. Continuous measurement over this interval could therefore resolve, at best,

$$
\delta x \approx \frac{1}{\sqrt{8 \Delta t \eta \kappa}},
$$

with a signal-to-noise ratio of one. We would like to achieve resolution comparable to the size of the quantum ground state, $x_{0}=\sqrt{\hbar / 2 m \omega}$, in some time interval which for now we will consider to be of the order of a mechanical oscillation 
$\Delta t=1 / \omega$, to outpace a realistic thermal-heating rate,

$$
\delta x_{\omega}=\sqrt{\frac{\omega}{8 \eta \kappa}} \equiv x_{0} .
$$

From this, we can conclude that in order to approach groundstate cooling sufficiently quickly, it is necessary for $\kappa / x_{0}^{2} \sim$ $\omega / 8 \eta$. This places a lower bound on the necessary measurement strength, with the trade-off for going to higher values being greater back-action heating and stochastic drift. Even idealized measurements introduce energy in the form of squeezing the state variances for any finite $\kappa$. Actively counteracting the disturbance caused by a probe light relies on efficiently gathering as much useful information as possible from every scattered photon. This, along with the necessary resolution requirement, is the criteria for a suitable measurement.

We can now assess the merits and shortcomings of various measurement techniques. Cameralike imaging has been used in previous experiments with particles in low-frequency traps. A camera follows a particle's position in a plane perpendicular to the direction of light being scattered from it. However, it is light scattered parallel to this plane that imparts the most recoil to the visible motion of the particle. This translates to a very low quantum efficiency. For example, $15 \%$ lightcollection efficiency from a radiating point dipole, $f(\theta)=$ $3 / 4 \cos ^{2}(\theta)$, translates to detecting $\sim 1 \%$ of the imparted recoil in the imaging plane. Meanwhile, a measurement of a particle's motion parallel to the light being scattered, with the same collection efficiency, translates to detecting $\sim 19 \%$ of the relevant recoil (as in Fig. 1). Even so, imaging is simple to implement and, for the purpose of initially damping the position variance to around a fraction of a micron, low quantum efficiency will not be an issue. For comparison, a $0.1-\mu$ m-diameter diamond in a trap $\omega=2 \pi \times 100 \mathrm{~Hz}$ will only be quantum limited when approaching the ground-state variance of roughly $x_{0} \approx 0.1 \mathrm{~nm}$. Many high-efficiency measurements capable of resolving beyond optical-wavelength amplitude motion require the particle to already be tightly confined. In a large trap, this necessitates some initial cooling so that the particle does not move outside the range of these measurement techniques. Current experiments have cooled to submilliKelvin temperatures with a silicon microsphere in a magneto-gravitational trap [18]. Damping the variance further, to within a micron, corresponds to a temperature of about a microKelvin and will require improved isolation from classical heating sources. Overcoming these classical noise challenges will bring experiments to the point where they could operate in our proposed strong feedback cooling regime.

Introducing a cavity around the suspended particle is often a reliable way to improve light-collection efficiency. Homodyning light from a standing-wave cavity can be used to efficiently track the position of a particle; however, this necessarily introduces a dipole potential tied to the measurement strength and has its own associated challenges [19]. Sideband cooling with near-resonance light within a cavity has also been proposed as a useful aid in achieving groundstate cooling [20]. However, this would not be compatible with the efficient on-axis light collection available in magnetic traps and, under optimal conditions, stops being beneficial for cooling compared to active feedback alone when $\eta \sim$ 0.2. This level of efficiency would hopefully be surpassed in future experiments with enhanced directional scattering. A sensitive velocity measurement was proposed for ion cooling by exploiting electromagnetically induced transparency [21]. This phenomenon could be observed in a traveling wave cavity with a diamond containing an $\mathrm{NV}$ center; however, the velocity information would only be contained in the spontaneously emitted radiation from a necessarily weakly excited state. For a very massive particle, this would be an extremely weak measurement, $\kappa / x_{0}^{2} \ll \Gamma_{\text {th }}$, unable to suitably resolve the particle for damping on short timescales. As discussed in the model section, the most suitable method we have found involves measuring the amplitude modulation of a standing wave due to a particle's motion in front of a single mirror. This technique has been successfully demonstrated with trapped ions $[11,22]$ and has the potential to be very effective for monitoring magnetically levitated nanoscopic particles, when combined with initial cooling of the oscillation amplitude to around a single optical wavelength.

\section{B. State estimation}

It will be necessary to process the measurement signal in order to perform feedback cooling since it is not possible to achieve damping by making shifts in the system Hamiltonian proportional to the position alone. Using the equations of motion that describe the particle, combined with the measurement record, the full system state can be continuously estimated. This type of information processing can quickly converge on both the true position and momentum values of the particle, while updating the expected error in the estimation.

Using the master equation (8) and the fact that $d\langle c\rangle=$ $\operatorname{Tr}[c d \rho]$, we can find equations of motion for the relevant position and momentum moments to describe a Gaussian state undergoing measurement,

$$
\begin{gathered}
d\langle x\rangle=\frac{1}{m}\langle p\rangle d t+\sqrt{8 \eta \kappa} V_{x} d W, \\
d\langle p\rangle=-m \omega^{2}\langle x\rangle d t+\sqrt{8 \eta \kappa} C_{x p} d W, \\
\partial_{t} V_{x}=\frac{2}{m} C_{x p}-8 \eta \kappa V_{x}^{2}, \\
\partial_{t} V_{p}=-2 m \omega^{2} C_{x p}+2 \hbar^{2} \kappa-8 \eta \kappa C_{x p}^{2}, \\
\partial_{t} C_{x p}=\frac{1}{m} V_{p}-m \omega^{2} V_{x}-8 \eta \kappa V_{x} C_{x p},
\end{gathered}
$$

where $V_{x}$ and $V_{p}$ are the position and momentum variances, and $C_{x p}=(1 / 2)\left\langle[x, p]_{+}\right\rangle-\langle x\rangle\langle p\rangle$ is the symmetrized covariance. The stochastic increments here can be rewritten in terms of the measurement record $d I$, and the equations can be solved to estimate the particle's full motional state. The integration would need to be carried out in real time with time steps $\delta t$ much shorter than the damping timescale, e.g., $\delta t \ll 1 \mathrm{~ms}$ when attempting to overdamp a $\omega=2 \pi \times 100 \mathrm{~Hz}$ oscillator. The particle's motion is expected to look thermal when cooling starts and this provides a good guess for the particle's initial-state variances. The measurement process itself also drives any state towards looking Gaussian, ensuring 


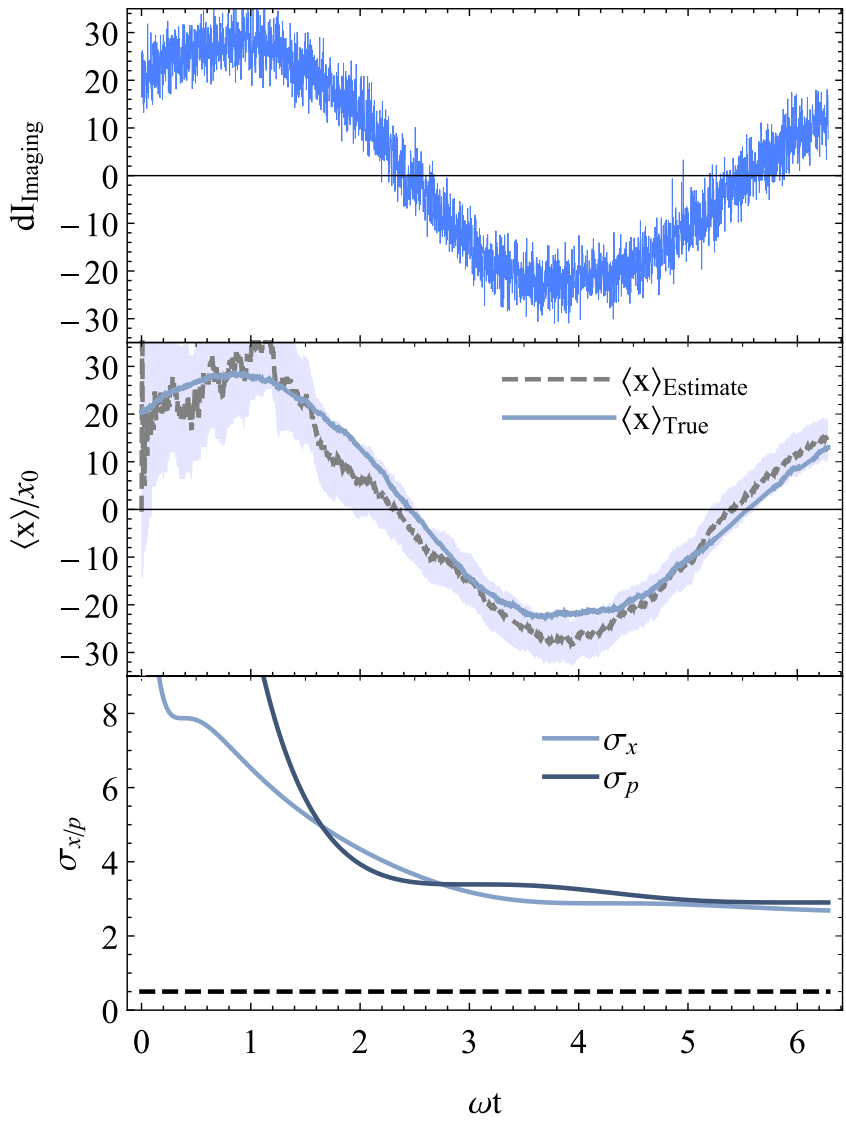

FIG. 2. Simulation of a trapped particle undergoing measurement, using (16)-(20). The normalized measurement strength, $\kappa x_{0}^{2} / \omega=1$, with $0.2 \%$ quantum efficiency, and an initial particle energy corresponding to a temperature of $T=1 \mu \mathrm{K}$. The top figure shows a numerically generated example of a position measurement and the middle figure shows the results of continuous-state estimation using the same signal. The estimated mean position plotted beside the true value and the shaded region covers 2 standard deviations in the estimate. The bottom figure shows the improvement in the standard deviation in both position (light line) and momentum (dark line) due to the measurement. The dashed line indicates the width of the motional ground state.

the continued reliability of these state equations. This procedure is not dissimilar to estimating the velocity by taking the derivative of the position signal, by passing it through a suitable band-pass filter. In fact, these state equations are exactly equivalent to the Kalman equations for a noisy classical system, and do indeed act like filters but with dynamic quality factors and cutoff frequencies. Kalman equations are designed to update information about a system based on a series of imperfect measurements and produce an estimate of the system that improves with time better than a series of measurements being made independently [23].

The effectiveness of estimating the state of a levitated particle over a single oscillation cycle is illustrated in Fig. 2 for a general position measurement. The true state is numerically modeled using the Gaussian moment equations (16)-(20), with an initial temperature of $1 \mu \mathrm{K}$, which might be realistically achieved with classical feedback damping. The stochastic measurement record (12) is also numerically

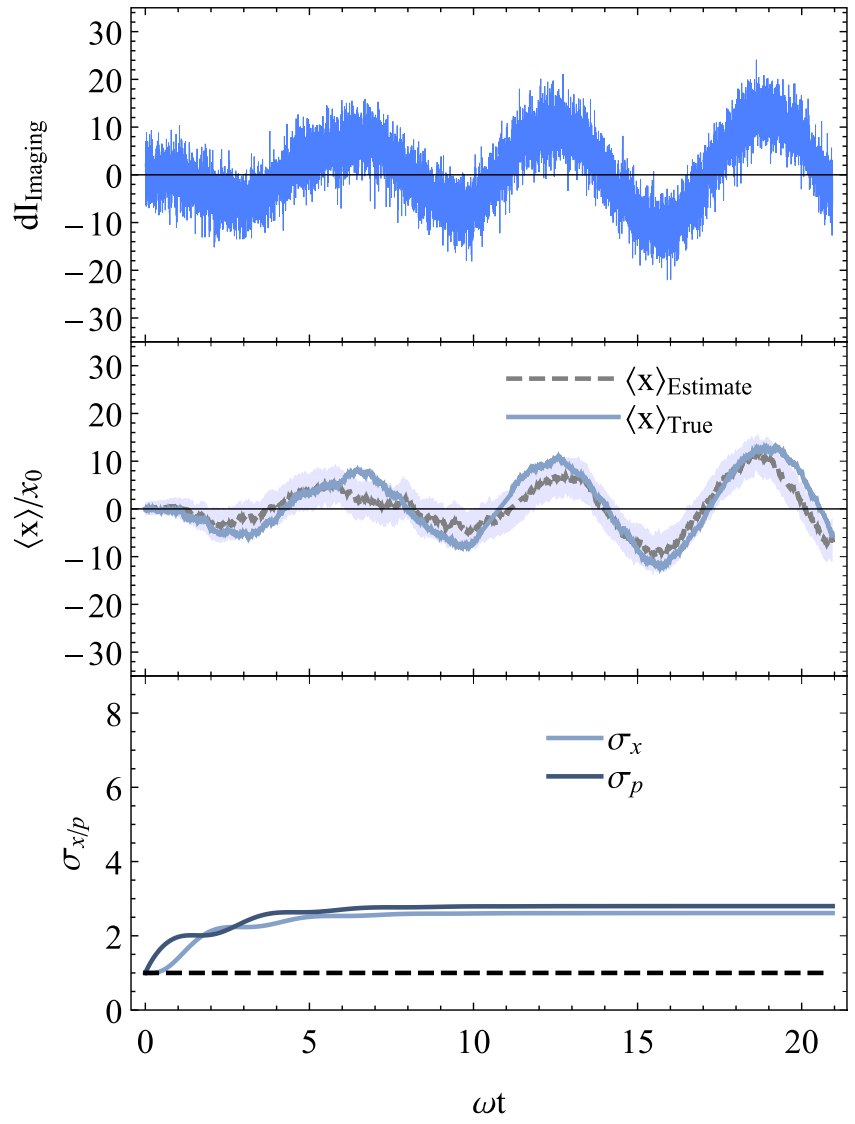

FIG. 3. Simulation of particle heating due to measurement over several oscillation cycles, using (16)-(20). The normalized measurement strength, $\kappa x_{0}^{2} / \omega=1$, with $0.2 \%$ quantum efficiency. The particle is initially in its ground state with temperature $T=0 \mathrm{~K}$. This figure is otherwise organized in the same way as Fig. 2.

generated based on the current true state. This is then used to update a second set of the same Gaussian moment equations to simulate the state estimation procedure. The state estimate is initiated with thermal variances, whereas the true state is modeled as a coherent state with thermal energy. The estimator quickly converges on the true state of the system, until reaching the resolution limit set by the measurement strength and quantum efficiency. This full-state model confirms the rough resolution limit (14). Quantum efficiency $\eta=0.02$ was chosen for the figure to show more pronounced lag in the estimator following the true quantum state.

The Gaussian state equations can also be used to illustrate the heating effects due to the measurement itself; see Fig. 3. For the considered measurement strengths, this is more easily visible with a state initially prepared at $T=0 \mathrm{~K}$. Without any other sources of environmental heating, the measurement will add energy into the system until it reaches a temperature associated with the magnitude of the photon shot noise. This temperature is higher with more intense illumination and presents a trade-off when trying to achieving a better resolution.

Full-state estimation could be implemented from room temperature, but is not initially necessary. If the particle can be confined and stably cooled to microKelvin temperatures, it 
makes sense to switch to full-state estimation when attempting to effectively overdamp the particle the rest of the way towards its quantum ground state. At higher temperatures, damping using the effective derivative of the position measurement via a simple band-pass filter should be sufficient.

\section{FEEDBACK COOLING}

There are two well-established approaches to applying feedback that take into account the effects of quantum noise: direct feedback of a force proportional to the measurement signal [10] and feedback based on real-time state estimation [12]. It is important to know whether feedback should be treated as direct in order to correctly account for how the noise in the measurement and in the system will be correlated. In the limit of instantaneous feedback, shot noise can be reintroduced from the measurement that directly correlates and interferes with the quantum noise driving the system evolution. Indirect feedback always filters out this noise in an intermediate step. The simplest approach to damping is to apply a force proportional and opposite to a particle's current velocity, and if measuring the velocity explicitly, this can be implemented as direct feedback [21]. Similarly, in the case of a high-quality oscillator, it is sufficient to feedback a signal proportional to the slowly varying momentum quadrature [17]. Both of these techniques require cooling over at least hundreds of oscillation cycles, which is not feasible in lowfrequency traps. In this case, indirect feedback using the state estimation is necessary, where the low trap frequencies will in fact be beneficial.

\section{A. Feedback procedure}

The optimal feedback strategy can be determined using classical control theory. In a classical system, there would not be noise fundamentally linked to the measurement strength, but this can be artificially enforced. This is useful because it allows well-developed control methods to be adapted for cooling $[19,24,25]$. Our sketch of the idea closely follows the work in Ref. [12].

For this system, it turns out not to be optimal to include the estimated state variances in the feedback function. They will be necessary to continuously solve for the mean position and momentum, but the feedback will not directly involve the variance values. The feedback Hamiltonian should simply be some linear function of the momentum and position operators scaled by functions of the estimated first-order moments,

$$
H_{f}=f(\langle x\rangle,\langle p\rangle) x+g(\langle x\rangle,\langle p\rangle) p .
$$

To find the appropriate form of the functions $f$ and $g$, we can define a cost function for the parameter we want to minimize, in this case the energy,

$$
C=\int_{0}^{t}\left[\operatorname{Tr}\left(\mathbf{x}^{T} P \mathbf{x} \rho\right)+q^{2} \mathbf{u}^{T} Q \mathbf{u}\right] .
$$

Here, $\mathbf{x}=\{x, p\}$ is the state vector and $\mathbf{u}=-K\langle\mathbf{x}\rangle$ is the feedback vector that we want to introduce in the dynamical equations for the mean moments (16),(17); the optimal form of the matrix $K$ is what needs to be determined. The matrices $P$ and $Q$ are chosen so that the cost function represents the system energy,

$$
P=Q=\left(\begin{array}{cc}
m \omega^{2} & 0 \\
0 & 1 / m
\end{array}\right) .
$$

The matrix $Q$ can be interpreted as accounting for an energy cost associated with the feedback. Including it in this way reflects a restriction on the magnitude of the feedback weighted by the parameter $q$, which will work out to be inversely proportional to the system damping rate.

Optimal feedback should attempt to localize both position and momentum simultaneously. To achieve this, the Hamiltonian should be of the form

$$
H_{f}=\frac{1}{q}(\langle p\rangle x+\langle x\rangle p),
$$

where we define $\Gamma=1 / q$ to be the system damping rate, and the parameter $q$ can be interpreted as a bound on the feedback response time. This accounts for the physical limitations of the feedback mechanism and places an upper bound on the optimal damping rate. For an infinitely broadband signal, $q \rightarrow 0$ and the damping rate could be arbitrarily high. With feedback, the new equations for the damped position and momentum are

$$
\begin{array}{r}
d\langle x\rangle=\frac{1}{m}\langle p\rangle d t+\sqrt{8 \eta \kappa} V_{x} d W-\Gamma\langle x\rangle, \\
d\langle p\rangle=-m \omega^{2}\langle x\rangle d t+\sqrt{8 \eta \kappa} C_{x p} d W-\Gamma\langle p\rangle .
\end{array}
$$

To directly damp the particle's velocity, a position term in the Hamiltonian can be introduced simply by using an externally applied force. When damping over many oscillation cycles, this also damps the position variance. To directly damp the position, however, requires a momentum term in the Hamiltonian.

One option to introduce this is with a time-dependent shift in the origin of the position coordinates, which in the rest frame of the trap manifests itself as a shift to the canonical momentum. The harmonic trap could be mechanically displaced or, equivalently, a constant force could be applied to the same effect,

$$
H^{\prime}=\frac{m \dot{x}^{\prime 2}}{2}+\frac{m \omega^{2}\left(x^{\prime}-v t\right)^{2}}{2},
$$

where we have defined $H^{\prime}$ to be the Hamiltonian in the laboratory frame and $v$ is the velocity at which the trap center is shifted. We need to shift coordinates and define a new canonical momentum to see this as position damping,

$$
\begin{gathered}
x=x^{\prime}-v t, \quad p=m(\dot{x}+v), \\
H^{\prime} \rightarrow H=\frac{p^{2}}{2 m}+\frac{m \omega^{2} x^{2}}{2}-\Gamma\langle x\rangle p,
\end{gathered}
$$

identifying $v=\Gamma\langle x\rangle$. Momentum damping could then be implemented with respect to these redefined coordinates,

$$
H=\frac{p^{2}}{2 m}+\frac{m \omega^{2} x^{2}}{2}-\Gamma\langle x\rangle p-\Gamma\langle p\rangle x,
$$

where the estimates of $\langle x\rangle$ and $\langle p\rangle$ are obtained by solving the state estimator equations (25),(26) using the position measurement $I$ shifted to the trap frame, e.g., $I \rightarrow(I-v t)=$ $\left(\left\langle x^{\prime}\right\rangle-v t\right)+\xi(t) / \sqrt{8 \kappa \eta}$. 


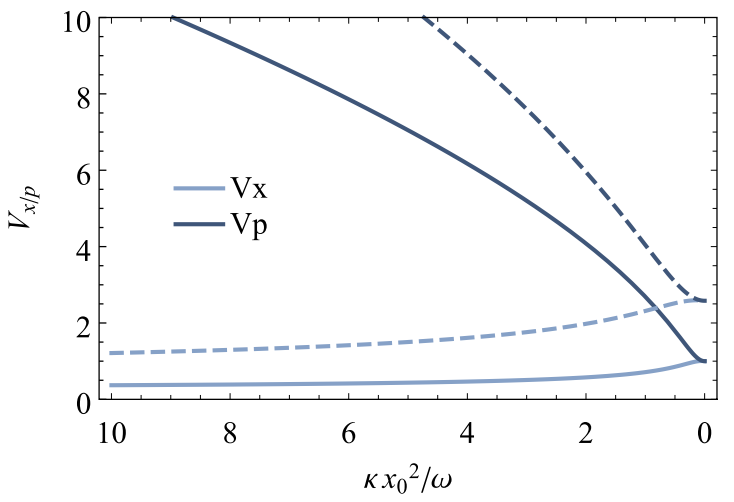

FIG. 4. Final resolution of the normalized position (light line) and momentum (dark line) variances of a trapped particle, from the steady-state solutions of a Gaussian estimator (32),(33). Variance values of less than 1 are squeezed compared to the harmonicoscillator ground state. The solid lines correspond to a measurement with perfect efficiency $\eta=1$ and the dashed lines $\eta=0.15$; these values and the measurement strength would vary depending on the nature of the measurement.

This produces optimal damping in the rest frame of the trap. Transformed back to the laboratory frame, with the momentum defined as $p^{\prime}=m \dot{x}^{\prime}$, the Hamiltonian now reads

$$
\begin{aligned}
H^{\prime} & =\frac{p^{\prime 2}}{2 m}+\frac{m \omega^{2}\left(x^{\prime}-v t\right)^{2}}{2}-\Gamma\langle p\rangle\left(x^{\prime}-v t\right) \\
& =\frac{p^{\prime 2}}{2 m}+\frac{m \omega^{2} x^{\prime 2}}{2}-m \omega^{2} \Gamma\langle x\rangle x^{\prime} t-\Gamma\langle p\rangle x^{\prime} .
\end{aligned}
$$

The shifts made to the trap center would have to be small, given the measurement's sensitivity to where the particle sits in the standing-wave field, but a piezoelectric device could be used to shake the trap in a controlled manner to achieve damping.

\section{B. Cooling results}

In this system, the introduction of linear feedback has no effect on the estimated variances conditioned on the measurement record. Their dynamics are governed by the measurement alone and we can therefore find the steady-state values for our feedback controlled state from the original equations for the Gaussian moments (18)-(20),

$$
\begin{aligned}
& \tilde{V}_{x}=\frac{2 m \omega}{\hbar} V_{x}=\left(\frac{2}{\eta} \frac{1}{\xi^{1 / 2}+1}\right)^{1 / 2}, \\
& \tilde{V}_{p}=\frac{2}{\hbar m \omega} V_{p}=\left(\frac{2}{\eta} \frac{\xi}{\xi^{1 / 2}+1}\right)^{1 / 2},
\end{aligned}
$$

where $\xi=1+16 \eta\left(\kappa x_{0}^{2} / \omega\right)^{2}$. These normalized variances are equal to one for a minimum uncertainty state. This is the case for unit efficiency and when the parameter $\xi \rightarrow 1$, which in turn is the case when the measurement strength $\kappa \rightarrow 0$. Relative to the trap frequency in optical traps, $\kappa$ is usually very small, but with a strong measurement $\kappa x_{0}^{2}>\omega$, the steadystate position variance is noticeably squeezed compared to the harmonic oscillator's natural ground state. Figure 4 shows how the conditional variances vary for the range of measurement strengths accessible in low-frequency magnetic traps.

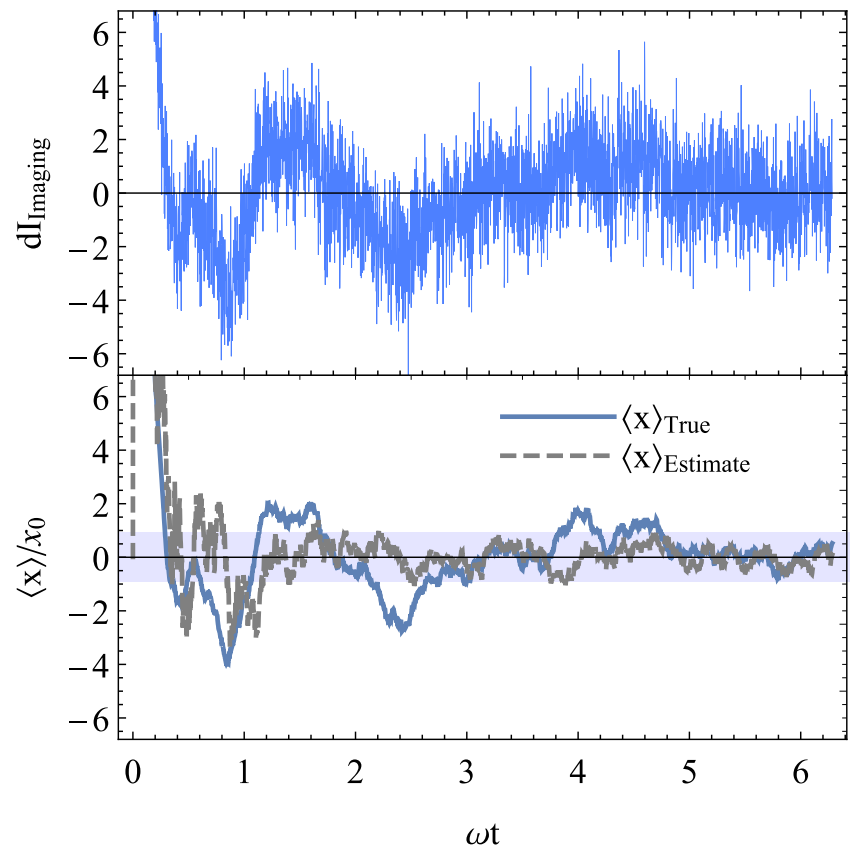

FIG. 5. Simulation of a damped levitated particle, using (25), (26), and (18)-(20). The normalized measurement strength, $\kappa x_{0}^{2} / \omega=$ 1 , with $15 \%$ quantum efficiency, and initial particle energy corresponding to a temperature of $T=1 \mu \mathrm{K}$. The top figure shows a numerically generated example of a position measurement. The bottom figure shows the evolution of the mean position of the true state alongside the estimated position from the measurement record. The estimated position is almost completely damped relative to the fundamental shot noise in the original measurement signal. The standard deviation of the true motion from $t=\pi / 2 \rightarrow 2 \pi$ is highlighted and matches the estimated variance (32).

The estimated variances are the best that could be resolved with a given measurement. We can then average over the measurement record to account for the excess variance due to the particle's motion. The applied feedback should limit this as much as possible, keeping the mean position and momentum values centered on zero. Using the equations for the mean position and momentum (25),(26) and following the rules of Ito calculus, we can calculate the excess variances, which we have distinguished with a superscript $E$,

$$
\begin{gathered}
\partial_{t} \tilde{V}_{x}^{E}=-2 \Gamma \tilde{V}_{x}^{E}+2 \omega \tilde{C}_{x p}^{E}+\frac{2 \omega}{\chi} \tilde{V}_{x}^{2}, \\
\partial_{t} \tilde{V}_{p}^{E}=-2 \Gamma \tilde{V}_{p}^{E}-2 \omega \tilde{C}_{x p}^{E}+\frac{2 \omega}{\chi} \tilde{C}_{x p}^{2}, \\
\partial_{t} \tilde{C}_{x p}^{E}=-2 \Gamma \tilde{C}_{x p}^{E}-\omega\left(\tilde{V}_{x}^{E}-\tilde{V}_{p}^{E}\right)+\frac{2 \omega}{\chi} \tilde{V}_{x} \tilde{C}_{x p} .
\end{gathered}
$$

The final state is always improved with stronger damping, which effectively counteracts the measurement shot noise, as well as removing the initial thermal energy. The return for increasing $\Gamma$ quickly drops of and, for moderate damping rates $\Gamma>\omega$, the steady-state variances approach the ideal limits given by the measurement resolution. This is reassuring since physically there would certainly be a bound to the feedback response time. Figure 5 shows a simulation of the feedback procedure for experimentally reasonable 
parameters $\eta=0.1, T_{\text {initial }}=1 \mu \mathrm{K}, k \omega / x_{0}^{2}=1, \Gamma=10$. The state is again modeled as a coherent state with thermal energy, and feedback is applied based on a numerically simulated state estimator. The particle's motion is almost completely damped after a single oscillation cycle and the excess variance in the mean position is highlighted, $\tilde{V}_{x}^{E} \sim 0.1$. The remaining motion is small compared to the fundamental resolution limit due to the photon shot noise.

From the steady-state expressions, we can also find the purity of the final state [26],

$$
\operatorname{Tr}\left(\rho^{2}\right)=(\hbar / 2)\left(V_{x} V_{p}-C_{x p}^{2}\right)^{-1 / 2} .
$$

If the damping is strong, the steady-state value is approximately that of a conditional state without any excess. With perfect detection, the final measured state looks pure and becomes increasingly mixed as the efficiency drops,

$$
P_{c}=\operatorname{Tr}\left(\rho_{c}^{2}\right)=\sqrt{\eta} .
$$

To reach the lowest temperatures, $\kappa$ would ideally be kept as low as possible to avoid squeezing due to the measurement. There is a balance then between resolving the particle fast enough to outpace environmental heating and wanting a weak probe to minimize squeezing. Notably, however, state purity has no dependence on the measurement strength, suggesting that the squeezed states with higher energy could reasonably be expected to have quantum properties which are just as visible.

The final average phonon number can be calculated using the combined conditional variances based on a particular measurement, and the excess variance seen when averaging over trajectories,

$$
\langle n\rangle=\frac{\left\langle x^{2}\right\rangle}{2}+\frac{\left\langle p^{2}\right\rangle}{2}-\frac{1}{2} .
$$

Steady-state phonon occupancy, calculated with (39), is shown in Fig. 6, for a range of measurement strengths and quantum efficiencies. These are the expected values that would be observed after damping, taking into account the estimated variance in the measurement signal (32),(33), and the excess variance associated with the remaining particle motion (34)-(36).

\section{CONCLUSIONS AND OUTLOOK}

In this article, we have analyzed processes for state estimation and feedback cooling of a low-frequency, magnetically levitated nanoparticle. Monitoring the particle's position through modulation of a standing wave in front of a mirror was chosen as the most suitable option, over monitoring the light output from a cavity. This should be relatively simple to integrate into current experiments and would allow for a high degree of variation in the measurement strength, which would be primarily dependent on the intensity of the probe beam. The need to damp both the particle momentum and position independently is likely to be the largest experimental difficulty after achieving sufficient isolation from environmental heating. The nature of the static magnets that make up these traps may make it possible to control the particle by dynamically shifting the trap center, and alternate methods

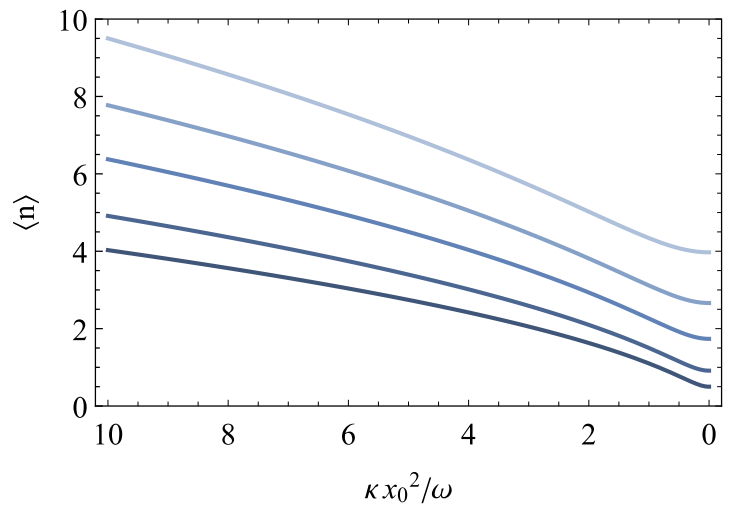

FIG. 6. Average steady-state phonon occupancy of a trapped nanoparticle after undergoing active feedback, calculated using the equations for a damped Gaussian state with excess noise (39). The effective damping rate (feedback gain) was chosen to be $\Gamma / \omega=$ 10 , strong enough to remove almost all stochastic drift due to the measurement disturbance. The quantum efficiencies from the top line down are $\eta=0.05,0.1,0.2,0.5,1.0$. The final occupancies range from $\langle n\rangle<3$, for currently feasible experimental parameters $(\eta=$ $0.2, k=1)$, to near zero, with perfect collection efficiency and a weaker measurement.

using a sequence of strong controlled laser pulses are also possible.

We suggest that measurement efficiency comparable to or greater than that achievable in ion traps, $\eta=0.15$, could realistically be reached in an experiment. Optimal feedback via state estimation with this level of efficiency could produce states competitively near the quantum ground state with some additional degree of squeezing, $\langle n\rangle<3$, with purity $P \approx 0.44$, in only a few oscillation periods. In current experiments, there are many factors to consider in order to extend the system reheating time, which will be the main barrier to achieving lower temperatures as it prevents the use of a less disruptive measurement probe. As these values improve and with the possibility of highly directional scattering for better collection efficiency, it may soon be feasible to reach below single-phonon occupancies using the methods outlined in this article. Most related experiments have so far assessed success based on a temperature associated with the measured motional power spectrum. Alternatively, there are recent proposals for distinguishing quantum motion via dynamical model selection solely using position measurements [27]. They look to identify quantum statistics from a series of position measurements after introducing a small perturbation to the trapping potential. The distinguishability is closely related to state purity, which should be safely within reach of the proposed cooling methods.

All of the methods discussed are applicable to submicronsized Rayleigh scatterers that can be effectively treated as point dipoles. High-quality nanodiamonds of this size have been produced for exactly the purpose of trapping and cooling [28]. Microscopic particles, on the other hand, would not usually be suitable for the subwavelength measurements suggested. However, large diamonds could still be cooled by tracking the position of pointlike NV impurities within them. 
Additionally, strong coupling between an NV spin and the mechanical oscillation of a nanodiamond can be engineered using a strong magnetic field gradient. There are proposals for generating low-number Fock states and possible spatial superposition states by manipulating a Jaynes-Cummings-type interaction Hamiltonian, in states prepared near the quantum ground state [3].

\section{ACKNOWLEDGMENTS}

The authors thank B. D'Urso, B. R. Slezak, C. W. Lewandowski, and P. Nachman for discussions, which motivated the present work, and for their helpful comments regarding experimental details. L.S.W. acknowledges support from the EPSRC Programme Grant DesOEQ (EP/P009565/1) and SUPA for a PECRE award to visit Montana State University.
[1] E. Verhagen, S. Deléglise, S. Weis, A. Schliesser, and T. J. Kippenberg, Nature (London) 482, 63 (2012).

[2] J. Chan, T. P. Alegre, A. H. Safavi-Naeini, J. T. Hill, A. Krause, S. Gröblacher, M. Aspelmeyer, and O. Painter, Nature (London) 478, 89 (2011).

[3] Z. Q. Yin, T. Li, X. Zhang, and L. M. Duan, Phys. Rev. A 88, 033614 (2013).

[4] A. A. Geraci, S. B. Papp, and J. Kitching, Phys. Rev. Lett. 105, 101101 (2010).

[5] A. Albrecht, A. Retzker, and M. B. Plenio, Phys. Rev. A 90, 033834 (2014).

[6] V. Jain, J. Gieseler, C. Moritz, C. Dellago, R. Quidant, and L. Novotny, Phys. Rev. Lett. 116, 243601 (2016).

[7] A. T. M. A. Rahman, A. C. Frangeskou, M. S. Kim, S. Bose, G. W. Morley, and P. F. Barker, Sci. Rept. 6, 21633 (2016).

[8] J.-F. Hsu, P. Ji, C. W. Lewandowski, and B. D'Urso, Sci. Rep. 6, 1 (2016).

[9] M. C. O'Brien, S. Dunn, J. E. Downes, and J. Twamley, Appl. Phys. Lett. 114, 053103 (2019).

[10] H. M. Wiseman and G. J. Milburn, Phys. Rev. A 49, 1350 (1994).

[11] P. Bushev, D. Rotter, A. Wilson, F. Dubin, C. Becher, J. Eschner, R. Blatt, V. Steixner, P. Rabl, and P. Zoller, Phys. Rev. Lett. 96, 043003 (2006).

[12] A. C. Doherty and K. Jacobs, Phys. Rev. A 60, 2700 (1999).

[13] K. Jacobs and D. A. Steck, Contemp. Phys. 47, 279 (2006).

[14] C. Gardiner and P. Zoller, Quantum Noise, Vol. 56 (Springer Science \& Business Media, Verlag, Berlin, Heidelberg, 2004).
[15] H. Carmichael, An Open Systems Approach to Quantum Optics, Vol. 18 (Springer Science \& Business Media, Verlag, Berlin, Heidelberg, 2009).

[16] H. M. Wiseman and G. J. Milburn, Phys. Rev. A 47, 1652 (1993).

[17] A. C. Doherty, A. Szorkovszky, G. I. Harris, and W. P. Bowen, Philos. Trans. R. Soc. A: Math. Phys. Eng. Sci. 370, 5338 (2012).

[18] B. R. Slezak, C. W. Lewandowski, J. Hsu, and B. D'urso, New J. Phys. 20, 063028 (2018).

[19] D. A. Steck, K. Jacobs, H. Mabuchi, S. Habib, and T. Bhattacharya, Phys. Rev. A 74, 012322 (2006).

[20] M. G. Genoni, L. Lami, and A. Serafini, Contemp. Phys. 57, 331 (2016).

[21] P. Rabl, V. Steixner, and P. Zoller, Phys. Rev. A 72, 043823 (2005).

[22] V. Steixner, P. Rabl, and P. Zoller, Phys. Rev. A 72, 043826 (2005).

[23] O. L. R. Jacobs, Introduction to Control Theory, Oxford Science Publications (Oxford University Press, 1993).

[24] D. A. Steck, K. Jacobs, H. Mabuchi, T. Bhattacharya, and S. Habib, Phys. Rev. Lett. 92, 223004 (2004).

[25] A. C. Doherty, A. S. Parkins, S. M. Tan, and D. F. Walls, Phys. Rev. A 57, 4804 (1998).

[26] W. H. Zurek, S. Habib, and J. P. Paz, Phys. Rev. Lett. 70, 1187 (1993)

[27] J. F. Ralph, M. Toroš, S. Maskell, K. Jacobs, M. Rashid, A. J. Setter, and H. Ulbricht, Phys. Rev. A 98, 010102(R) (2018).

[28] A. C. Frangeskou, A. T. M. A. Rahman, L. Gines, S. Mandal, O. A. Williams, P. F. Barker, and G. W. Morley, New J. Phys. 20, 043016 (2018). 